\title{
Ultra-narrowband NIR Photomultiplication Organic Photodetectors based on Charge Injection Narrowing
}

\author{
Ming Liu ${ }^{\mathrm{a}}$, Jian Wang ${ }^{\mathrm{b}}$, Zijin Zhao ${ }^{\mathrm{a}}$, Kaixuan Yang ${ }^{\mathrm{a}}$, Pablo Durand ${ }^{\mathrm{c}}$, Fabien Ceugniet ${ }^{\mathrm{c}}$, Gilles \\ Ulrich $^{\mathrm{c}}$, Lianbin $\mathrm{Niu}^{\mathrm{d}}{ }^{*}$, Yao $\mathrm{Ma}^{\mathrm{e}}$, Nicolas Leclerc ${ }^{\mathrm{c} *}$,Xiaoling Ma ${ }^{\mathrm{a}}$, Liang Shen ${ }^{\mathrm{e}}$, Fujun Zhang ${ }^{\mathrm{a}}{ }^{*}$
}

a) Key Laboratory of Luminescence and Optical Information, Ministry of Education, Beijing Jiaotong University, 100044, Beijing, P. R. China

b) College of Physics and Electronic Engineering, Taishan University, Taian, 271021, P. R. China

c) Institut de Chimie et Procédés pour l'Energie, l'Environnement et la Santé (ICPEES), Université de Strasbourg, CNRS, UMR 7515, 25 rue Becquerel, 67087, Strasbourg, Cedex 02, France

d) College of Physics and Electronic Engineering, Chongqing Normal University, Chongqing, 401331, P. R. China

e) State Key Laboratory of Integrated Optoelectronics, College of Electronic Science and Engineering, Jilin University, 130012, Changchun, China

*Corresponding author: E-mail: niulb03@126.com (Lianbin), leclercn@unistra.fr (Nicolas), fjzhang@bjtu.edu.cn (Fujun)

\begin{abstract}
Ultra-narrowband NIR photomultiplication organic photodetectors (PM-OPDs) were realized in ITO/PEDOT:PSS/active layers/Al based on the interfacial-traps induced charge injection narrowing (CIN) concept. The rather less Bod Ethex-Hex (BEH) is imbedded in polymer donor matrix to form some isolated electron traps. The trapped electrons in $\mathrm{BEH}$ closing $\mathrm{Al}$ electrode will force interfacial band-bending for hole-tunneling-injection, resulting in the photomultiplication. The PM-OPDs with P3HT:BEH as active layers exhibit narrow response peak at $850 \mathrm{~nm}$ with full width at half-maximum (FWHM) of $27 \mathrm{~nm}$, as well as a rather weak response from $650 \mathrm{~m}$ to $800 \mathrm{~nm}$. The rejection ratios (RRs) are 11 at $\mathrm{EQE}_{855 \mathrm{~nm}} / \mathrm{EQE}_{700 \mathrm{~nm}}$ and 10 at $\mathrm{EQE}_{855 \mathrm{~nm}} / \mathrm{EQE}_{750 \mathrm{~nm}}$, respectively. The $\mathrm{EQE}$ of $29,700 \%$ at $850 \mathrm{~nm}$ was achieved in the PM-OPDs by incorporating 0.02 wt $\% \mathrm{~F}_{6} \mathrm{TCNNQ}$ under $-13 \mathrm{~V}$ bias. An EQE of $15,300 \%$ at 850 $\mathrm{nm}$ was achieved in the ternary PM-OPDs at $-13 \mathrm{~V}$ bias, with the markedly enhanced RRs of 44 at $\mathrm{EQE}_{855 \mathrm{~nm}} / \mathrm{EQE}_{700 \mathrm{~nm}}$ and 30 at $\mathrm{EQE}_{855 \mathrm{~nm}} / \mathrm{EQE}_{750 \mathrm{~nm}}$. This work provides a smart strategy to achieve highly efficient ultra-narrowband NIR PM-OPDs by adjusting trapped-electron-distribution near hole injection electrode and molecular arrangement for
\end{abstract}


enhancing hole mobility in active layers.

Keyword: Organic photodetectors, Photomultiplication, Narrowband, Tunneling injection

\section{Introduction}

Photodetectors have attracted wide interest due to their potential in different application fields with the distinct spectral response range, such as image sensing, surveillance, fluorescence microscopy, and defense applications. ${ }^{[1-5]}$ Broadband response organic photodetectors can be commonly achieved by employing one wide bandgap material and one narrow bandgap material as the active layers, also working under photodiode model with external quantum efficiency (EQE) less than $100 \% .{ }^{[6-12]}$ Narrowband response organic photodetectors are more challenging to engineer because of the relatively broad photon harvesting range of organic materials, especially for photomultiplication organic photodetectors (PM-OPDs) with EQE larger than $100 \%{ }^{[13-16]}$ The PM-OPDs can be realized through interfacial trap-induced charge tunneling injection, the spectral response range of PM-OPDs is determined by the trapped-charge-distribution near the opposite charge injection electrode, which provides more possibilities to adjust response range of PM-OPDs. ${ }^{[17-19]}$ The PM-OPDs with PFN instead of PEDOT:PSS as anode interfacial layer can work well under forward and reverse bias, exhibiting broadband response from $350 \mathrm{~nm}$ to $800 \mathrm{~nm}$ and narrowband response peak at $800 \mathrm{~nm}$ with full width at half-maximum (FWHM) of $40 \mathrm{~nm} .{ }^{[20]}$ The PM-OPDs possessing broadband/narrowband photodetection capability were realized by using $16 \mathrm{~nm} \mathrm{Al}$ as the top semitransparent electrode. ${ }^{[21]}$ Although the EQE of PM-OPDs can be markedly improved along with reverse bias increase, it is great challenge to realize high detection sensitivity of PM-OPDs under low bias due to the relatively low charge mobility of organic semiconducting materials. ${ }^{[22-23]}$ In fact, charge mobility will also influence the response speed of organic photodetectors, especially for thick-film devices.

Table 1. The type works on narrowband organic photodetectors.

\begin{tabular}{cccccc}
\hline Active layer & Strategy & $\begin{array}{c}\text { FWHM } \\
(\mathrm{nm})\end{array}$ & $\begin{array}{c}\lambda \text { max } \\
(\mathrm{nm})\end{array}$ & $\begin{array}{c}\text { EQE/bias } \\
(\mathrm{V})\end{array}$ & Ref. \\
\hline PCDTBT:PC 71 BM & $\mathrm{CCN}$ & 65 & 680 & $18 \% /-1 \mathrm{~V}$ & {$[24]$} \\
$\mathrm{MAPbI}_{2} \mathrm{Br}$ & $\mathrm{CCN}$ & 80 & 650 & $10 \% /-0.5 \mathrm{~V}$ & {$[25]$} \\
$\mathrm{MAPbBr}_{3}$ & $\mathrm{CCN}$ & 20 & 570 & $3 \% /-4 \mathrm{~V}$ & {$[26]$}
\end{tabular}




\begin{tabular}{|c|c|c|c|c|c|}
\hline P3HT:NT812:Y6 & EDN & 50 & 860 & $60 \% /-0.1 \mathrm{~V}$ & [27] \\
\hline P3HT:PC 60 BM:CdTe & $\mathrm{CCN}$ & 100 & 660 & $200 \% /-6 \mathrm{~V}$ & [28] \\
\hline P3HT:PC 71 BM & CIN & 27 & 650 & $13800 \% /-40 \mathrm{~V}$ & [29] \\
\hline P3HT:PTB7-Th:PC ${ }_{71}$ BM & CIN & 30 & 800 & $2000 \% /-50 \mathrm{~V}$ & [21] \\
\hline P3HT:PTB7-Th:BEH & CIN & 27 & 850 & $15300 \% /-13 \mathrm{~V}$ & This work \\
\hline
\end{tabular}

Some smart strategies were proposed to prepare narrowband organic photodetectors without optical filter, such as charge injection narrowing (CIN) based on bulk heterojunction device structure, charge collection narrowing (CCN) also based on bulk heterojunction device structure and exciton dissociation narrowing $(E D N)$ based on planar heterojunction device structure. ${ }^{[21,24-30]}$ The typical works of narrowband organic photodetectors are summarized in the Table $\mathbf{1}$. According to the working mechanism of organic photodetectors based on CCN and EDN concept, the EQE values are less than $100 \%$ at the price of charge carrier loss and exciton loss, respectively. A current amplifier is necessary in the application of organic photodetectors working under photodiode model to abstract light signal current from the large noise background during weak light detection. Meredith et al. firstly reported narrowband organic photodetectors by manipulating internal quantum efficiency based on $\mathrm{CCN}$ concept, charge collection efficiency is distinct for different wavelength light due to different penetration depth in thick active layers. ${ }^{[2]}$ The CCN concept was successfully realized in perovskite photodetectors with narrowband response. ${ }^{[25]}$ Huang et al. firstly realized narrowband organic photodetectors by manipulating exciton dissociation interface in planar heterojunction device based on the EDN concept. ${ }^{[27]}$ Zhang et al. firstly proposed PM-OPDs with efficient electron or hole transport channels and the opposite charge isolated traps within the thick bulk heterojunction active layers based on CIN concept. The narrowband PM-OPDs were prepared by using $2.5 \mu \mathrm{m} \mathrm{P} 3 \mathrm{HT}: \mathrm{PC}_{71} \mathrm{BM}(100: 1, \mathrm{wt} / \mathrm{wt})$ as the active layers, exhibiting a rapidly increased EQE from 600\% @ - 20 V to 33,600\% @ -50 V bias, also keeping the FWHM of EQE spectra less than $30 \mathrm{~nm}$ under different bias. ${ }^{[29]}$ The spectral response of PM-OPDs based on CIN concept is determined by absorption edge of thick active layers, which can be well explained by the trapped-charge-distribution near injection electrode according to the Lambert-Beer law. ${ }^{[24,28]}$ It is a great challenge to achieve high EQE of narrowband PM-OPDs 
under low bias, especially for response range in near infrared (NIR) region.

In this work, ultra-narrowband NIR PM-OPDs were achieved with the sandwich structure of ITO/PEDOT:PSS/active layers $(3 \mu \mathrm{m}) / \mathrm{Al}$, polymer materials P3HT and PTB7-Th were selected as donor and a small BODIPY-based molecule, named Bod Ethex-Hex (BEH), was used as non-fullerene acceptor. The photogenerated electrons will be trapped in the isolated BEH surrounded by the polymers. Recently, the use of small doping molecules has grown considerably, either in thermoelectricity [Influence of dopant size and doping method on the structure and thermoelectric properties of PBTTT films doped with F6TCNNQ and F4TCNQ, V. Vijayakumar, $P$. Durand, H. Zeng, V. Untilova, L. Herrmann, P. Algayer, N. Leclerc and M. Brinkmann, Journal of Materials Chemistry C, 2020, doi.org/10.1039/D0TC02828B], organic solar cells [Y. Lin, M. I. Nugraha, Y. Firdaus, A. D. Scaccabarozzi, F. Aniés, A.-H. Emwas, E. Yengel, X. Zheng, J. Liu, W. Wahyudi, E. Yarali, H. Faber, O. M. Bakr, L. Tsetseris, M. Heeney and T. D. Anthopoulos, ACS Energy Lett. 2020, 5, 3663-3671] or organic field-effect transistors [Paterson, A. F.; Singh, S.; Fallon, K. J.; Hodsden, T.; Han, Y.; Schroeder, B. C.; Bronstein, H.; Heeney, M.; McCulloch, I.; Anthopoulos, T. D. Recent Progress in High-Mobility Organic Transistors: A Reality Check. Adv. Mater. 2018, 30 (36), 1801079]. In this work, we report for the first time, such a strategy applied to PM-OPDs, by using the small molecular material F6TCNNQ as solid additive to optimize both polymer molecular arrangement and hole transport within the active layer. The chemical structures, lowest unoccupied molecular orbital (LUMO) and the highest occupied molecular orbital (HOMO) levels, absorption spectra of P3HT, PTB7-Th, BEH and $\mathrm{F}_{6}$ TCNNQ, as well as the schematic device configuration of PM-OPDs are illustrated in Fig. 1. The PM-OPDs with P3HT:BEH (100:3, $\mathrm{wt} / \mathrm{wt}$ ) as the active layers exhibit two narrow spectral response from $650 \mathrm{~nm}$ to $800 \mathrm{~nm}$ and from $800 \mathrm{~nm}$ to $900 \mathrm{~nm}$. It is apparent that the rather less (0.02 wt\%) $\mathrm{F}_{6}$ TCNNQ incorporation plays key role in enhancing EQE values of PM-OPDs. The EQE values of PM-OPDs with $\mathrm{F}_{6} \mathrm{TCNNQ}$ reach $29,700 \%$ at $850 \mathrm{~nm}$ under $-13 \mathrm{~V}$ bias, which is one order larger than $2,600 \%$ at $850 \mathrm{~nm}$ of PM-OPDs without $\mathrm{F}_{6} \mathrm{TCNNQ}$ under $-40 \mathrm{~V}$ bias. To optimize response range and improve rejection ratios (RRs) of narrowband PM-OPDs, polymer PTB7-Th was incorporated to adjust the trapped-electron-distribution near Al electrode. The ternary PM-OPDs with P3HT:PTB7-Th:BEH (100:0.5:3, wt/wt) as active layers exhibit a narrow response range from $830 \mathrm{~nm}$ to $900 \mathrm{~nm}$ with the FWHM of $27 \mathrm{~nm}$ under different reverse bias. The optimal ternary PM-OPDs exhibit an EQE 
of $15,300 \%$ at $850 \mathrm{~nm}$ under $-13 \mathrm{~V}$ bias, the RRs of the optimal ternary PM-OPDs are 44 at EQE850 nm/EQE700 $\mathrm{nm}$ and 30 at EQE850 nm/EQE750 nm. This work provides a simple and efficient strategy to prepare ultra-narrowband NIR PM-OPDs with rapid response speed.

(a) a)

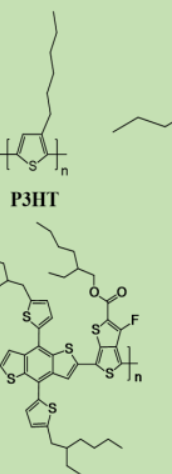

PTB7-Th

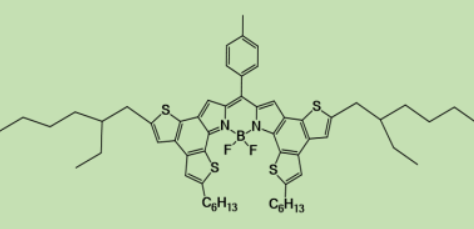

Bod Ethex-Hex (BEH)

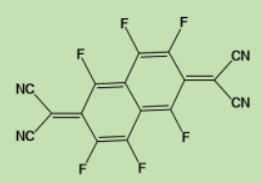

F $_{6}$ TCNNQ

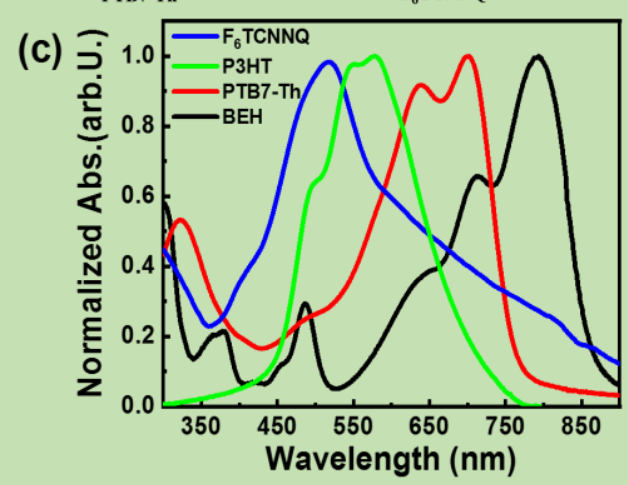

(b)

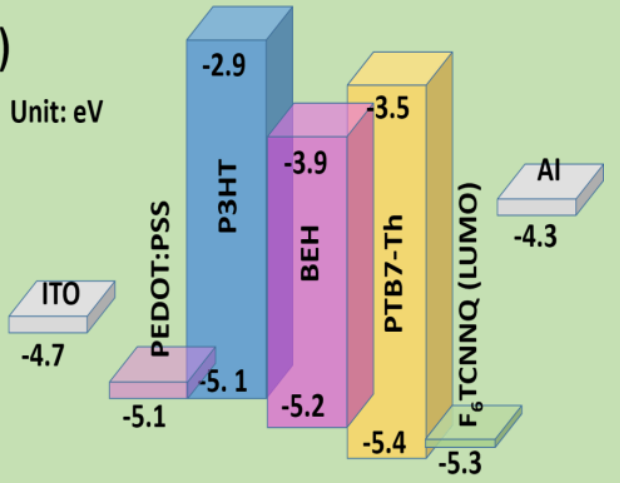

(d)

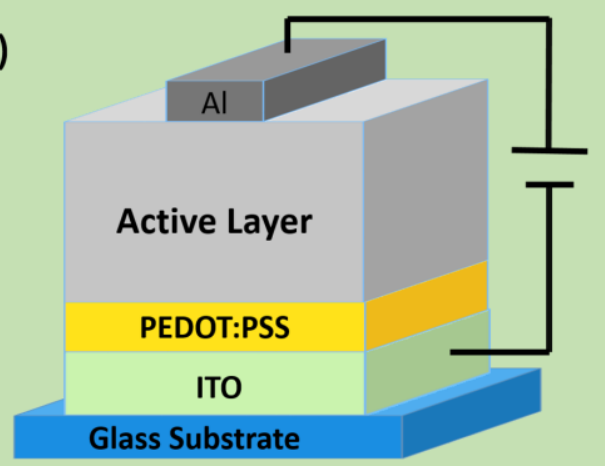

Figure 1. (a) Chemical structures of P3HT, PTB7-Th, BEH and $\mathrm{F}_{6}$ TCNNQ. (b) Schematic energy levels diagram of P3HT, PTB7-Th, BEH and $\mathrm{F}_{6}$ TCNNQ. (c) The normalized UV-Vis-NIR absorption spectra of pure $\mathrm{F}_{6} \mathrm{TCNNQ}$, P3HT, PTB7-Th and BEH films. (d) Device configuration diagram of our prepared PM-OPDs.

\section{Results and discussion}

Series of PM-OPDs were fabricated with P3HT:BEH as active layers, the weight ratios of P3HT to BEH was adjusted from 100:1 to 100:7 (wt/wt) in the blend solutions. Some isolated electron traps of P3HT/BEH/P3HT will be formed due to the different LUMO levels of P3HT and BEH, the isolated trap density can be altered by finely adjusting BEH content. The detailed current density versus voltage $(J-V)$ curves of all PM-OPDs in dark and under $2 \mathrm{~mW} / \mathrm{cm}^{2}$ white light illumination are exhibited in Fig. S1. The optimal weight ratio of P3HT:BEH is about 100:3 (wt/wt) by comprehensively evaluating the current density in dark $\left(J_{D}\right)$ and current density under light conditions $\left(J_{L}\right)$ of the corresponding PM-OPDs. To further improve the performance of PM-OPDs, $\mathrm{F}_{6}$ TCNNQ dopant was incorporated into the blend solutions to prepare active layers. 
All the active layers were rinsed by spin coating alcohol to remove superficial $F_{6}$ TCNNQ, which can restrain hole direct injection from $\mathrm{Al}$ electrode in dark condition to keep the low $J_{D}$ of PM-OPDs. The LUMO levels of $\mathrm{F}_{6} \mathrm{TCNNQ}$ is $-5.3 \mathrm{eV}$, measured by using the cyclic voltammetry (CV) method (see in Fig. S2). The deep LUMO level of F $_{6}$ TCNNQ can trap more electrons in active layers. The higher amount of trapped electrons near $\mathrm{Al}$ electrode is expected to induce a greater bending of interfacial band and consequently increase the hole-tunneling-injection efficiency. The $J-V$ curves of PM-OPDs without and with $\mathrm{F}_{6}$ TCNNQ in active layers were measured in dark and under $2 \mathrm{~mW} / \mathrm{cm}^{2}$ white light illumination conditions, as exhibited in Fig. 2a. It is apparent that PM-OPDs with $\mathrm{F}_{6} \mathrm{TCNNQ}$ exhibit the markedly increased $J_{L}$ and slightly increased $J_{D}$ in comparisons with those of PM-OPDs without $\mathrm{F}_{6}$ TCNNQ under the same biases, indicating that the $\mathrm{F}_{6}$ TCNNQ-doped PM-OPDs possess stronger photon detection ability. The EQE spectra of PM-OPDs without and with $\mathrm{F}_{6} \mathrm{TCNNQ}$ were measured under different reverse bias, as exhibited in Fig. 2b and 2c. Both PM-OPDs exhibit two narrow spectral response from $650 \mathrm{~nm}$ to $800 \mathrm{~nm}$ and from $800 \mathrm{~nm}$ to $900 \mathrm{~nm}$. The ultra-narrow response of PM-OPDs can be well explained by CIN concept, which is mainly determined by trapped-electron-distribution near $\mathrm{Al}$ electrode. According to the Lambert-Beer law, ${ }^{[24,28]}$ less incident photon can transmit through thick active layer to $\mathrm{Al}$ electrode, especially in the strong absorption range of the active layer. The absorption spectra of thick active layers with and without $\mathrm{F}_{6} \mathrm{TCNNQ}$ were measured and are inserted in Fig. 2b and 2c. The incident photon with wavelength less than $650 \mathrm{~nm}$ can be completely harvested by thick active layers, resulting in almost no photogenerated electrons trapped in $\mathrm{BEH}$ near $\mathrm{Al}$ electrode. The absent trapped electrons in $\mathrm{BEH}$ near $\mathrm{Al}$ electrode can not induce interfacial-band-bending for hole-tunneling-injection, resulting in the EQE values closing to zero in this spectral range. The response range of the PM-OPDs locates at the absorption edge of thick active layers because some incident photons can transmit through thick active layer to generate some trapped electrons in $\mathrm{BEH}$ near $\mathrm{Al}$ electrode. This phenomenon can be well explained according to the CIN concept. ${ }^{[31-34]}$ The slightly different EQE spectra of PM-OPDs with or without $\mathrm{F}_{6}$ TCNNQ can be observed in the spectral range from $700 \mathrm{~nm}$ to $830 \mathrm{~nm}$, as exhibited in Fig. 2d, which is mainly attributed to the distinct photon harvesting ability in this spectral range. The EQE values of all PM-OPDs are enhanced along with the reverse bias increase, especially for the PM-OPDs with $\mathrm{F}_{6}$ TCNNQ. The EQE values of PM-OPDs with $\mathrm{F}_{6}$ TCNNQ is 
approaching to $29,700 \%$ under $-13 \mathrm{~V}$ bias, which is one order larger than $2,600 \%$ of PM-OPDs without $\mathrm{F}_{6} \mathrm{TCNNQ}$ under $-40 \mathrm{~V}$ bias. According to the working mechanism of PM-OPDs, the EQE value of PM-OPDs is mainly determined by the number of charge carrier flowing through the device per unit. The relatively large EQE values of PM-OPDs with $\mathrm{F}_{6} \mathrm{TCNNQ}$ should be attributed to the enhanced hole mobility in the corresponding active layers.
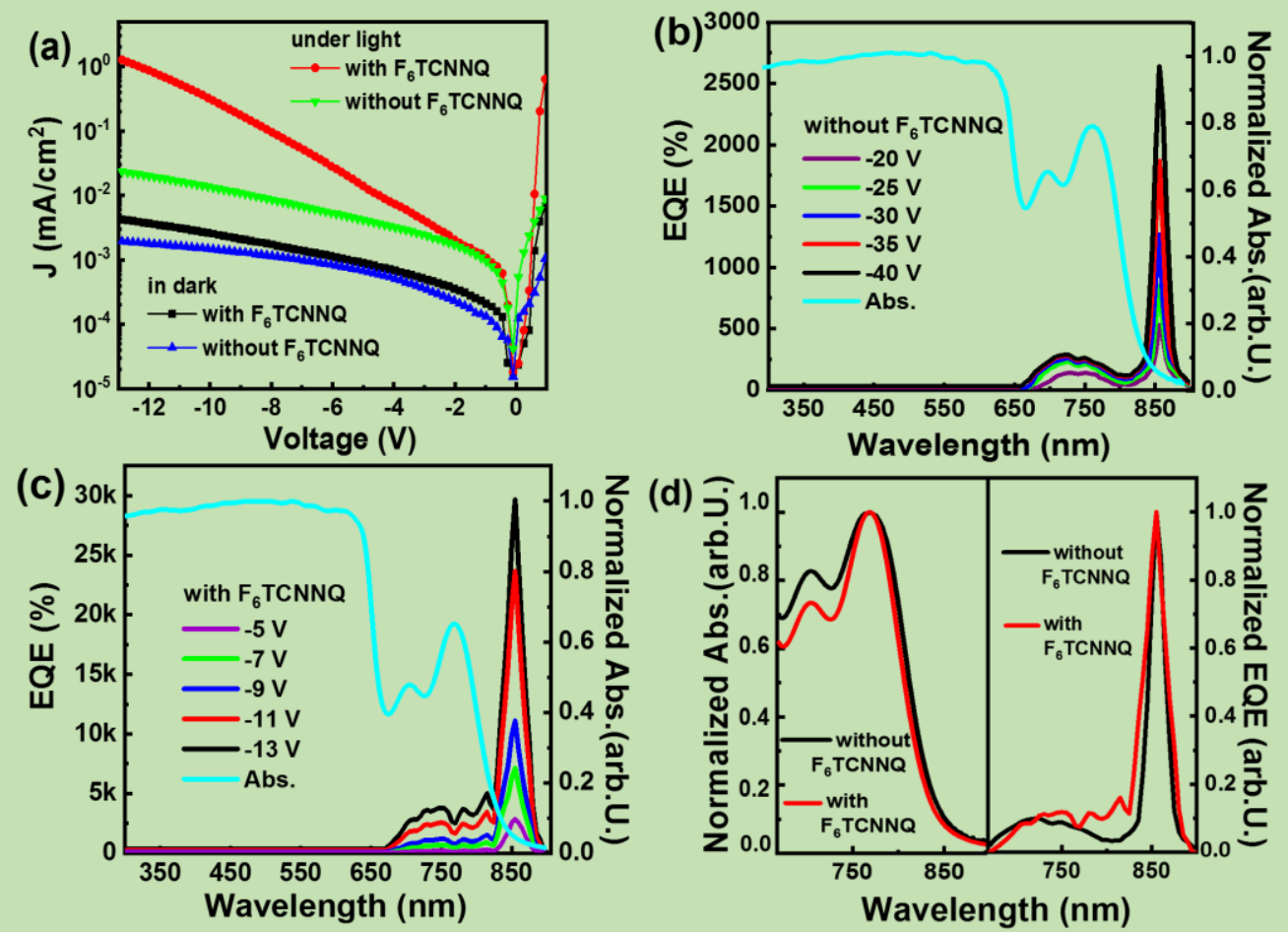

Figure 2. (a) The $J-V$ curves of the PM-OPDs with P3HT:BEH (100:3, wt/wt) as active layers in dark condition and under $2 \mathrm{~mW} / \mathrm{cm}^{2}$ white light illumination. (b) The EQE spectra and normalized absorption spectrum of PM-OPDs without $\mathrm{F}_{6} \mathrm{TCNNQ}$. (c) The EQE spectra and normalized absorption spectrum of PM-OPDs with $0.02 \mathrm{wt} \% \mathrm{~F}_{6} \mathrm{TCNNQ}$. (d) Normalized absorption spectra and normalized EQE spectra.

In order to investigate the effect of $\mathrm{F}_{6} \mathrm{TCNNQ}$ incorporation on hole mobility in active layers, hole-only devices were prepared with the device structure of ITO/PEDOT:PSS/active layers/Au. According to the In $\left(\mathrm{d}^{3} / \mathrm{V}^{2}\right)-(\mathrm{V} / \mathrm{d})^{0.5}$ curves of PM-OPDs with or without $\mathrm{F}_{6} \mathrm{TCNNQ}$ shown in Fig. 3a, hole mobility are $1.94 \times 10^{-5} \mathrm{~cm}^{2} \mathrm{v}^{-1} \mathrm{~s}^{-1}$ and $1.07 \times 10^{-6} \mathrm{~cm}^{2} \mathrm{v}^{-1} \mathrm{~s}^{-1}$ in the active layers with and without $\mathrm{F}_{6} \mathrm{TCNNQ}$, respectively. The enhanced hole mobility leads to an increase of charge carriers flowing through the device per unit, resulting in larger $J_{L}$ and EQE values. The transient photocurrent of PM-OPDs were investigated to further confirm hole mobility in active layers with and without $\mathrm{F}_{6} \mathrm{TCNNQ}$, as exhibited in Fig. 3b. The $850 \mathrm{~nm}$ light with intensity of $7 \mathrm{~mW} \mathrm{~cm}$ 
was modulated by an electronic-shutter with $10 \mathrm{~s}$ period. The response times of PM-OPDs without and with $\mathrm{F}_{6} \mathrm{TCNNQ}$ are $624 \mathrm{~ms}$ and $358 \mathrm{~ms}$, respectively. The rise processes of all PM-OPDs without $\mathrm{F}_{6} \mathrm{TCNNQ}$ are obviously slower than that of PM-OPDs with $\mathrm{F}_{6} \mathrm{TCNNQ}$ when the excitation light is turned on. The increased photocurrent of PM-OPDs is likely to originate from the hole tunneling injection due to the injection barrier narrowing induced by trapped electrons in $\mathrm{BEH}$ near $\mathrm{Al}$ electrode. The slow rise processes of PM-OPDs without $\mathrm{F}_{6} \mathrm{TCNNQ}$ can be explained by the low hole mobility, resulting in the less injected holes flowing through the active layer and collected by ITO electrode per unit. For $\mathrm{F}_{6} \mathrm{TCNNQ}$-doped PM-OPDs, the rapid rise processes can be observed when the excitation light is turned on, indicating a larger amount of injected holes flowing through the active layer and collected by the ITO electrode per unit. This is due to the enhanced hole mobility measured in the active layer. Most of the trapped electrons will be released or recombined with injected holes after excitation light is turned off, resulting in the photocurrent falling down to the initial state.
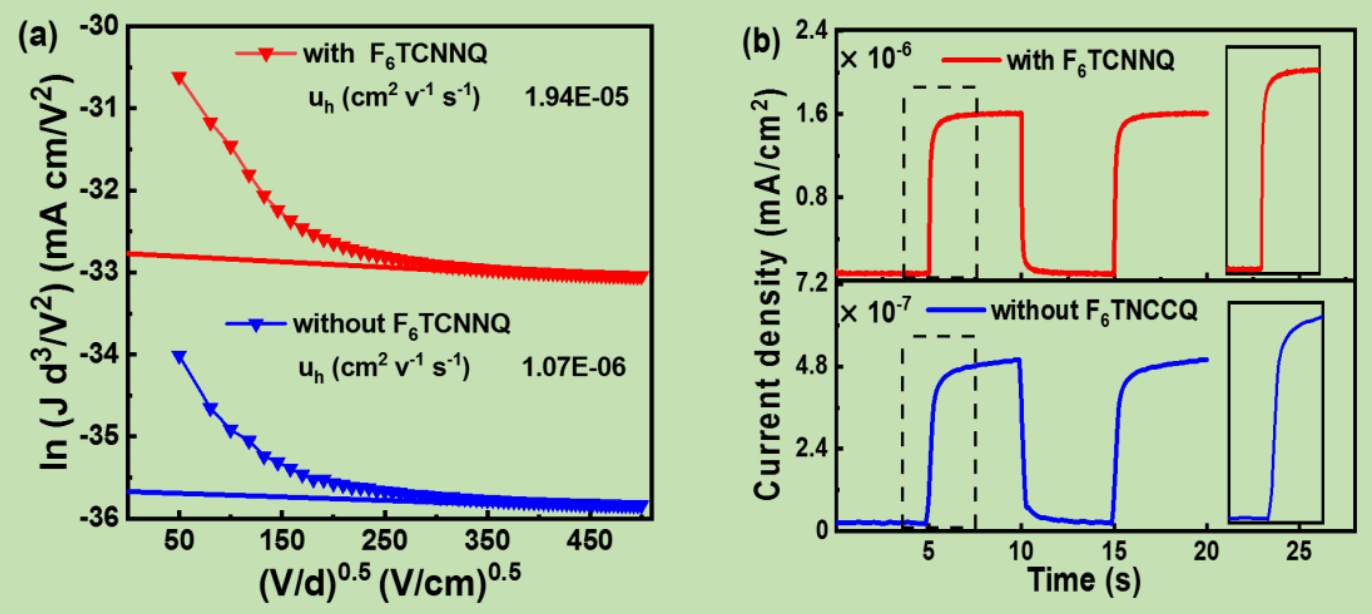

Figure 3. (a) In $\left(d^{3} / \mathrm{V}^{2}\right)-(\mathrm{V} / \mathrm{d})^{0.5}$ of hole-only devices in dark condition. (b) Transient current density of the PM-OPDs under $850 \mathrm{~nm}$ illumination with an intensity of $7 \mathrm{~mW} \mathrm{~cm}^{-2}$ condition.

It is well known that charge mobility in the active layers should be strongly impacted by the (macro-)molecular arrangement of blend films. ${ }^{[35-40]}$ In order to further investigate the effect of $\mathrm{F}_{6}$ TCNNQ on molecular arrangement in the active layers, out-of-plane (OOP) and in-plane (IP) line-cuts of blend films were investigated by grazing incidence X-ray diffraction, as exhibited in Fig. 4. The blend films with $\mathrm{F}_{6} \mathrm{TCNNQ}$ exhibit distinct (100) peak and weak (010) peak in OOP and IP direction, which indicates relatively high crystallinity degree of P3HT in blend films with 
$\mathrm{F}_{6} \mathrm{TCNNQ}$. The enhanced diffraction peak intensity in IP direction indicates a face-on preferential orientation of $\mathrm{P} 3 \mathrm{HT}$ in blend films with $\mathrm{F}_{6} \mathrm{TCNNQ}$. The preferential face-on orientation of P3HT is beneficial to hole transport along the perpendicular direction of substrate, ${ }^{[41-43]}$ which can well explain the enhanced response speed and increased EQE of PM-OPDs with $\mathrm{F}_{6} \mathrm{TCNNQ}$.
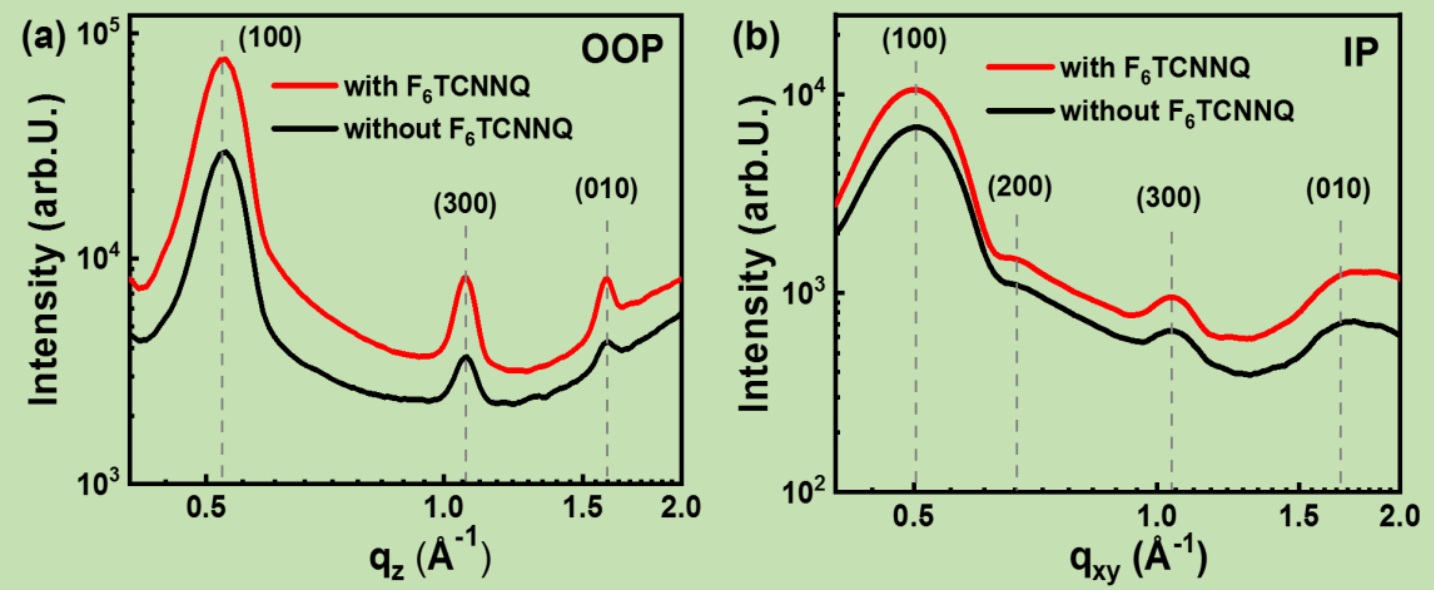

Figure 4. The (a) OOP and (b) IP line-cuts of P3HT:BEH blend films with and without $\mathrm{F}_{6} \mathrm{TCNNQ}$.

To further narrow spectra response range of PM-OPDs, narrow bandgap material PTB7-Th was selected as the third component to prepare ternary PM-OPDs with P3HT:PTB7-Th:BEH $(100: 0.5: 3, w t / w t)$ as active layers. The EQE spectra of ternary PM-OPDs under different reverse bias can be investigated, as exhibited in Fig. 4a. It is apparent that the spectra response of ternary PM-OPDs can be efficiently restrained in the $650 \mathrm{~nm}$ to $830 \mathrm{~nm}$ compared with that of PM-OPDs without PTB7-Th, which can be explained by the normalized absorption spectra of blend films with or without PTB7-Th, as inserted in the Fig. 4a. Only one ultra-narrow response from $830 \mathrm{~nm}$ to $900 \mathrm{~nm}$ can be well kept in the ternary PM-OPDs with the FWHM of $27 \mathrm{~nm}$, which can be well explained by the slightly enhanced photon harvesting from $650 \mathrm{~nm}$ to $830 \mathrm{~nm}$ of ternary active layers. The EQE values of ternary PM-OPDs are increased along with the increase of reverse bias and reach $15,300 \%$ at $850 \mathrm{~nm}$ under $-13 \mathrm{~V}$ bias. To accurately evaluate the noise equivalent power (NEP) and the specific detectivity ( $\left.\mathrm{D}^{*}\right)$ of optimal PM-OPDs, the total noise current $\left(i_{n}\right)$ was directly measured from the fast Fourier transform (FFT) of dark current as a function of time under $-13 \mathrm{~V}$ bias, as shown in Fig. $\mathbf{4 b}$. It is observed that the $i_{n}$ of the optimal PM-OPDs depends on the low frequency ( $\mathrm{f}<50 \mathrm{~Hz}$ ), indicating that $i_{n}$ is dominated by $1 / f$ noise in the low frequency 
range ( $\mathrm{f}<50 \mathrm{~Hz}$ ). The shot noise current $\left(\mathrm{i}_{\mathrm{s}}\right)$ is decided by dark current and can be calculated by the Equation: $i_{\mathrm{s}}=\sqrt{2 e I_{D} B}$, as inserted in Fig. $4 \mathbf{b}$. Obviously, the lower value of $i_{\mathrm{s}}$ compared with that of $i_{n}$ indicates that noise of optimal PM-OPDs is determined by $i_{n}$.
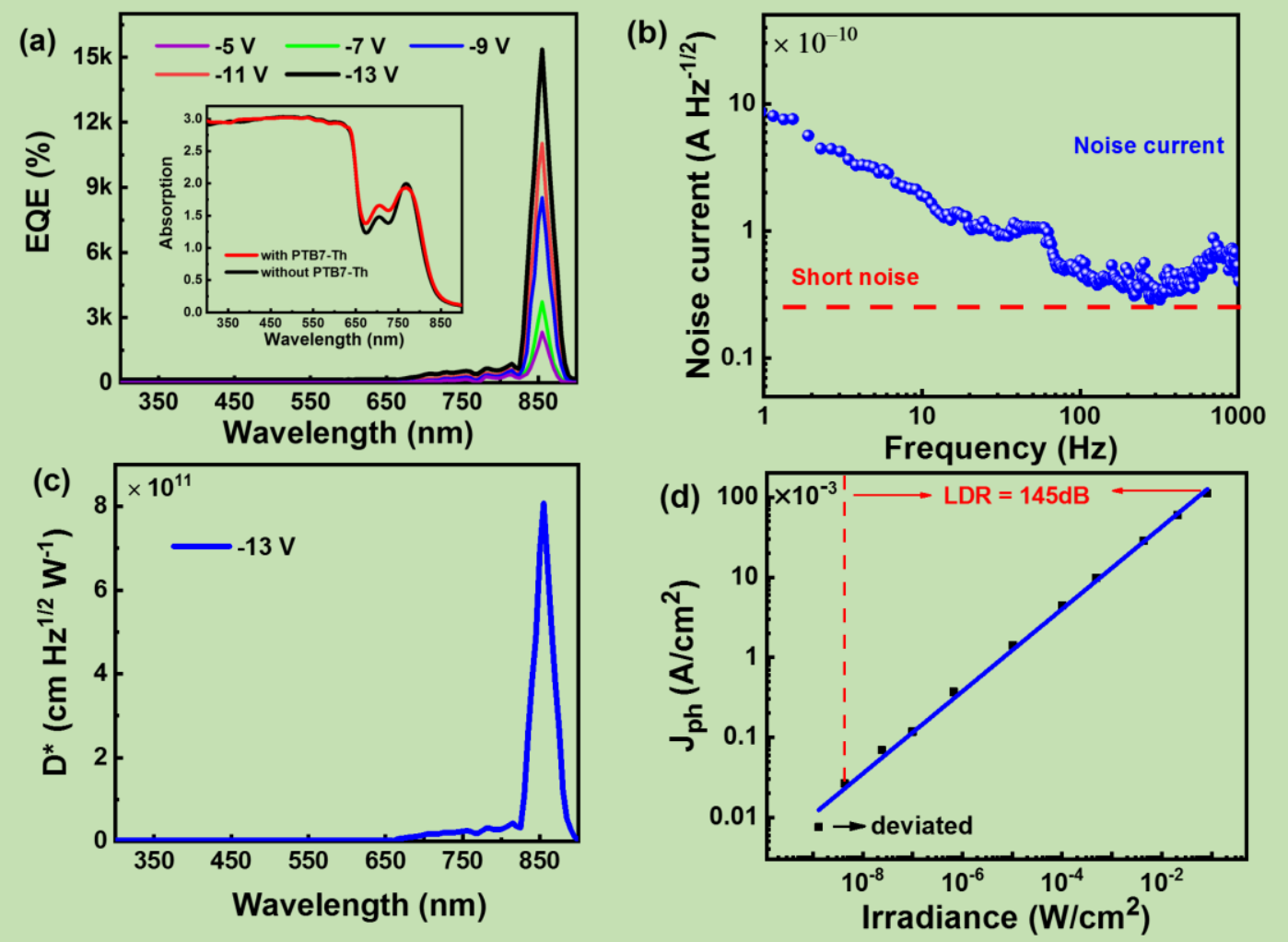

Figure 5. (a) The EQE spectra of the ternary PM-OPDs. (b) The total noise current and shot noise current of the ternary PM-OPDs under $-13 \mathrm{~V}$ bias. (c) The corresponding $\mathrm{D}^{*}$ spectrum of the ternary PM-OPDs under $-13 \mathrm{~V}$ bias. (d) The LDR of the ternary PM-OPDs measured at $-13 \mathrm{~V}$ bias.

The noise equivalent power (NEP) and specific detectivity (D*) of the PM-OPDs should be evaluated according to the following equations with the measured $i_{n}$ and EQE conditions: ${ }^{[44-47]}$

$$
\begin{aligned}
& N E P=\frac{i_{n}}{R \sqrt{B}}\left(W H z^{-1 / 2}\right) \\
& D^{*}=\frac{\sqrt{A}}{N E P}\left(\mathrm{~cm} \mathrm{~Hz} z^{1 / 2} W^{-1}\right)
\end{aligned}
$$

where R is the responsivity and is exhibited in Fig. $\mathbf{S 3}, \mathrm{i}_{\mathrm{n}}$ is total noise current, B is the electrical bandwidth, NEP is the noise equivalent power, and $\mathrm{A}$ is the active area. The $\mathrm{i}_{\mathrm{n}}$ of ternary PM-OPDs is about $2.53 \times 10^{-13} \mathrm{~A} \mathrm{~Hz}^{-1 / 2}$. The NEP spectrum of the ternary PM-OPDs under $-13 \mathrm{~V}$ is shown in Fig. S4 and the lowest NEP of the ternary PM-OPDs is $2.40 \times 10^{-13} \mathrm{~W} \mathrm{~Hz}^{-1 / 2}$ at $850 \mathrm{~nm}$. 
The D* of the ternary PM-OPDs was calculated based on the NEP and is shown in Fig. 4c. The D* approaches $8.8 \times 10^{11} \mathrm{~cm} \mathrm{~Hz}^{1 / 2} \mathrm{~W}^{-1}$ under $-13 \mathrm{~V}$ bias. The LDR as the crucial parameter of PM-OPDs represents that the responsivity can be kept constant under different incident light powers. ${ }^{[31,48]}$ The LDR of the ternary PM-OPDs was measured and arrives to $145 \mathrm{~dB}$ at $-13 \mathrm{~V}$ bias, as shown in Fig. 4d. When incident light intensity is lower than $404 \mathrm{pW} / \mathrm{cm}^{2}$, the linearity of the ternary PM-OPDs is lost because the lower amount of photogenerated electrons trapped in BEH near $\mathrm{Al}$ electrode, can not induce interfacial-band-bending for hole-tunneling-injection from external circuit.

\section{Conclusion}

In summary, ultra-narrowband NIR PM-OPDs were prepared with the sandwich structure of ITO/PEDOT:PSS/active layers $(3 \mu \mathrm{m}) / \mathrm{Al}$, the working mechanism of narrowband is interfacial-trapped charge induced injection narrowing, obeying the CIN concept. The optimal PM-OPDs with $\mathrm{F}_{6}$ TCNNQ exhibit high EQE of $29,700 \%$ at $850 \mathrm{~nm}$ due to efficient hole tunneling injection and enhanced hole transport in the active layers. The $\mathrm{F}_{6}$ TCNNQ dopant plays key role in optimizing polymer molecular arrangement, increasing hole mobility and inducing the interfacial band more bending for efficient hole tunneling injection. The PM-OPDs with P3HT:BEH (100:3, $\mathrm{wt} / \mathrm{wt}$ ) as thick active layers exhibit two narrowband spectral response from $650 \mathrm{~nm}$ to $800 \mathrm{~nm}$ and from $800 \mathrm{~nm}$ to $900 \mathrm{~nm}$. The narrowband response of ternary PM-OPDs can be further optimized by incorporating PTB7-Th as the third component, exhibiting one ultra-narrow response range from $830 \mathrm{~nm}$ to $900 \mathrm{~nm}$ with FWHM of $27 \mathrm{~nm}$ under forward bias. The ternary PM-OPDs exhibit EQE of $15,300 \%$ and maximum $\mathrm{D}^{*}$ of $8.8 \times 10^{11} \mathrm{~cm} \mathrm{~Hz}^{1 / 2} \mathrm{~W}^{-1}$ under $-13 \mathrm{~V}$ bias, as well as obviously enhanced RRs compared with PM-OPDs with P3HT:BEH (100:3, wt/wt) as active layers. This work provides an efficient strategy to achieve ultra-narrowband NIR PM-OPDs.

\section{Supporting Information}

Supporting Information is available from the Wiley Online Library or from the author.

\section{Acknowledgements}

This work was supported by the National Natural Science Foundation of China (61975006, 61874016, 62075155), Postdoctoral Innovative Talent Support Program (BX20200042), the Beijing Natural Science Foundation (4192049). Pablo Durand and Nicolas Leclerc would like to 
thanks the Region Grand Est and the French National Research Agency (ANR) for their financial support (Anisotherm project; ANR-17-CE05-0012).

\section{Conflict of interest}

The authors declare no competing financial interests.

\section{References}

[1] S. O. Kelley, C. A. Mirkin, D. R. Walt, R. F. Ismagilov, M. Toner, E. H. Sargent, Nat. Nanotechnol. 2014, 9, 969-980.

[2] L. Zhou, R. Wang, C. Yao, X. Li, C. Wang, X. Zhang, C. Xu, A. Zeng, D. Zhao, F. Zhang, Nat. Commun. 2015, 6, 6938.

[3] K. J. Baeg, M. Binda, D. Natali, M. Caironi, Y. Y. Noh, Adv. Mater. 2013, 25, 4267-4295.

[4] L. Gao, K. Zeng, J. Guo, C. Ge, J. Du, Y. Zhao, C. Chen, H. Deng, Y. He, H. Song, G. Niu, J. Tang, Nano Lett. 2016, 16, 7446-7454.

[5] R. D. Jansen-van Vuuren, A. Armin, A. K. Pandey, P. L. Burn, P. Meredith, Adv. Mater. 2016, $28,4766-4802$.

[6] Q. An, J. Wang, X. Ma, J. Gao, Z. Hu, B. Liu, H. Sun, X. Guo, X. Zhang, F. Zhang, Energy Environ. Sci. 2020, DOI: 10.1039/d0ee02516j.

[7] X. Ma, Z. Xiao, Q. An, M. Zhang, Z. Hu, J. Wang, L. Ding, F. Zhang, J. Mater. Chem. A 2018, $6,21485-21492$.

[8] J. Gao, W. Gao, X. Ma, Z. Hu, C. Xu, X. Wang, Q. An, C. Yang, X. Zhang, F. Zhang, Energy Environ. Sci. 2020, 13, 958-967.

[9] Z. Hu, L. Yang, W. Gao, J. Gao, C. Xu, X. Zhang, Z. Wang, W. Tang, C. Yang, F. Zhang, Small 2020, 16, 2000441.

[10] J. Gao, J. Wang, Q. An, X. Ma, Z. Hu, C. Xu, X. Zhang, F. Zhang, Sci. China: Chem. 2019, 63, 83-91.

[11]C. Xu, H. Chen, Z. Zhao, J. Gao, X. Ma, S. Lu, X. Zhang, Z. Xiao, F. Zhang, J. Energy Chem. 2020, DOI: 10.1016/j.jechem.2020.09.025.

[12] M. Zhang, R. Ming, W. Gao, Q. An, X. Ma, Z. Hu, C. Yang, F. Zhang, Nano Energy 2019, 59, $58-65$.

[13] Z. Zhao, C. Xu, L. Niu, X. Zhang, F. Zhang, Laser Photonics Rev. 2020, 14, 2000262.

[14] K. Yang, J. Wang, J. Miao, J. Zhang, F. Zhang, J. Mater. Chem. C 2019, 7, 9633-9640. 
[15] W. Wang, F. Zhang, H. Bai, L. Li, M. Gao, M. Zhang, X. Zhan, Nanoscale 2016, 8, 5578-5586.

[16] Z. Zhao, J. Wang, C. Xu, K. Yang, F. Zhao, K. Wang, X. Zhang, F. Zhang, J. Phys. Chem. Lett. 2020, 11, 366-373.

[17] A. Pierre, I. Deckman, P. B. Lechene, A. C. Arias, Adv. Mater. 2015, 27, 6411-6417.

[18] Z. Zhao, J. Wang, J. Miao, F. Zhang, Org. Electron. 2019, 69, 354-360.

[19] L. Li, F. Zhang, J. Wang, Q. An, Q. Sun, W. Wang, J. Zhang, F. Teng, Sci. Rep. 2015, 5, 9181.

[20] J. Miao, F. Zhang, M. Du, W. Wang, Y. Fang, Adv. Opt. Mater. 2018, 6, 1800001.

[21] W. Wang, M. Du, M. Zhang, J. Miao, Y. Fang, F. Zhang, Adv. Opt. Mater. 2018, 6, 1800249.

[22] Y. Wang, A. T.-R. Tan, T. Mori, T. Michinobu, J. Mater. Chem. C 2018, 6, 3593-3603.

[23] B. Maiti, A. Schubert, S. Sarkar, S. Bhandari, K. Wang, Z. Li, E. Geva, R. J. Twieg, B. D. Dunietz, Chem. Sci. 2017, 8, 6947-6953.

[24] A. Armin, R. D. Jansen-van Vuuren, N. Kopidakis, P. L. Burn, P. Meredith, Nat. Commun. 2015, 6, 6343.

[25] Q. Lin, A. Armin, P. L. Burn, P. Meredith, Nat. Photonics 2015, 9, 687-694.

[26] Y. Fang, Q. Dong, Y. Shao, Y. Yuan, J. Huang, Nat. Photonics 2015, 9, 679-686.

[27] B. Xie, R. Xie, K. Zhang, Q. Yin, Z. Hu, G. Yu, F. Huang, Y. Cao, Nat. Commun. 2020, 11, 2871.

[28] L. Shen, Y. Fang, H. Wei, Y. Yuan, J. Huang, Adv. Mater. 2016, 28, 2043-2048.

[29] W. Wang, F. Zhang, M. Du, L. Li, M. Zhang, K. Wang, Y. Wang, B. Hu, Y. Fang, J. Huang, Nano Lett. 2017, 17, 1995-2002.

[30] W. Wang, D. Zhao, F. Zhang, L. Li, M. Du, C. Wang, Y. Yu, Q. Huang, M. Zhang, L. Li, J. Miao, Z. Lou, G. Shen, Y. Fang, Y. Yan, Adv. Funct. Mater. 2017, 27, 1703953.

[31] S. Yoon, K. M. Sim, D. S. Chung, ACS Nano 2019, 13, 2127-2135.

[32] M. Liu, J. Miao, J. Wang, Z. Zhao, K. Yang, X. Zhang, H. Peng, F. Zhang, J. Mater. Chem C 2020, 8, 9854-9860.

[33] J. Miao, M. Du, Y. Fang, X. Zhang, F. Zhang, Sci. China: Chem. 2019, 62, 1619-1624.

[34] J. Miao, F. Zhang, Laser Photonics Rev. 2019, 13, 1800204.

[35] Q. An, X. Ma, J. Gao, F. Zhang, Sci. Bull. 2019, 64, 504-506. 
[36] X. Ma, Q. An, O. A. Ibraikulov, P. Lévêque, T. Heiser, N. Leclerc, X. Zhang, F. Zhang, J. Mater. Chem. A 2020, 8, 1265-1272.

[37] J. Wang, X. Ma, J. Wang, R. Ming, Q. An, J. Zhang, C. Yang, F. Zhang, Small 2019, 15, 1902602.

[38] Z. Hu, Z. Wang, F. Zhang, J. Mater. Chem. A 2019, 7, 7025-7032.

[39] C. Xu, J. Wang, Z. Zhao, J. Gao, Z. Hu, X. Ma, X. Zhang, F. Zhang, Sustain. Energy Fuels 2020, 4, 3979-3984.

[40] J. Gao, J. Wang, C. Xu, Z. Hu, X. Ma, X. Zhang, L. Niu, J. Zhang, F. Zhang, Sol. RRL 2020, 4, 20000364.

[41] Z. Hu, J. Wang, X. Ma, J. Gao, C. Xu, K. Yang, Z. Wang, J. Zhang, F. Zhang, Nano Energy 2020, 78, 105376.

[42] C. Xu, J. Wang, Q. An, X. Ma, Z. Hu, J. Gao, J. Zhang, F. Zhang, Nano Energy 2019, 66, 104116.

[43] X. Ma, J. Wang, J. Gao, Z. Hu, C. Xu, X. Zhang, F. Zhang, Adv. Energy Mater. 2020, 10, 2001404.

[44] J. Miao, M. Du, Y. Fang, F. Zhang, Nanoscale 2019, 11, 16406-16413.

[45] K. Yang, J. Wang, Z. Zhao, F. Zhao, K. Wang, X. Zhang, F. Zhang, Org. Electron. 2020, 83, 105739.

[46] D. Yang, D. Ma, Adv. Opt. Mater. 2019, 7, 1800522.

[47] J. Liu, H. Wen, L. Shen, Nanotechnology 2020, 31, 214001.

[48] Z. Ren, J. Sun, H. Li, P. Mao, Y. Wei, X. Zhong, J. Hu, S. Yang, J. Wang, Adv. Mater. 2017, 29,1702055 .

\section{Table of Contents}

\section{Ultra-narrowband NIR Photomultiplication Organic Photodetectors based on}

\section{Charge Injection Narrowing}

Ming Liu, Jian Wang, Zijin Zhao, Kaixuan Yang, Pablo Durand, Fabien Ceugniet, Gilles Ulrich, Lianbin Niu*, Nicolas Leclerc*,Xiaoling Ma, Fujun Zhang* 


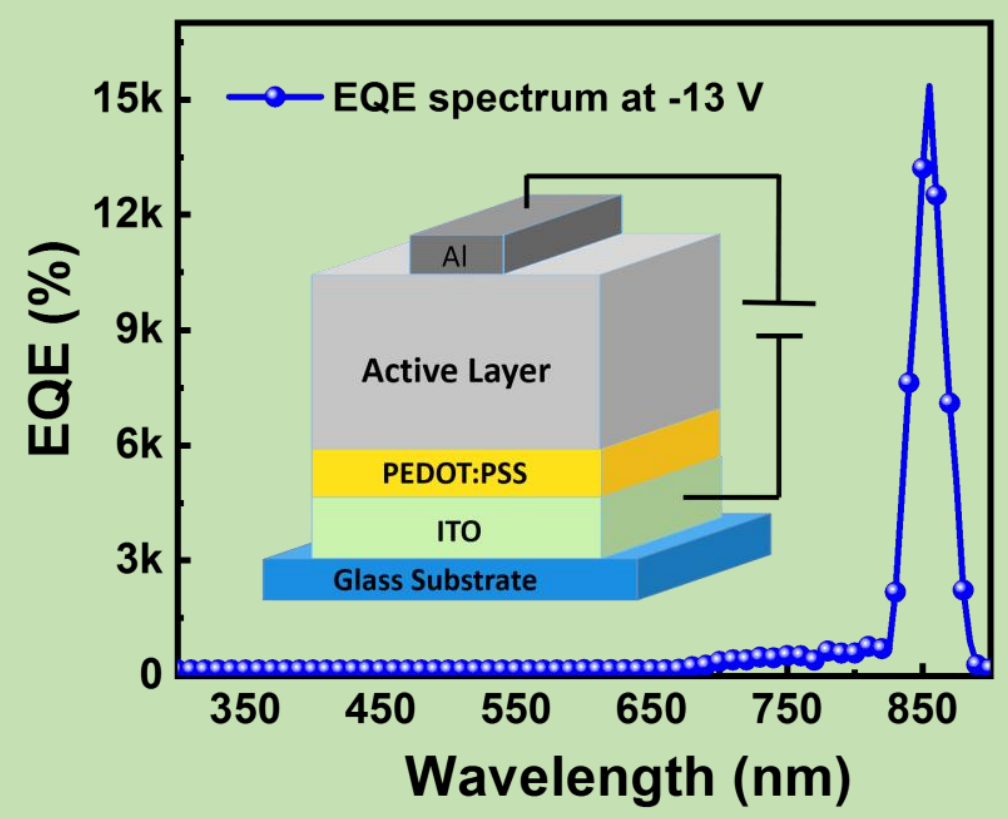

Ultra-narrowband NIR PM-OPDs were realized with P3HT:PTB7-Th:BEH (100:0.5:3, wt/wt) as active layers by incorporating $0.02 \mathrm{wt} \% \mathrm{~F}_{6} \mathrm{TCNNQ}$, exhibiting EQE of $15,300 \%$ at $850 \mathrm{~nm}$ with FWHM of $27 \mathrm{~nm}$ under $-13 \mathrm{~V}$ bias. The $\mathrm{F}_{6}$ TCNNQ dopant plays key role in optimizing polymer molecular arrangement for efficient hole transport. 